\title{
Weighted estimates for vector-valued multilinear square function
}

Wenjuan Li and Manli Song*

\author{
"Correspondence: \\ mlsong@nwpu.edu.cn \\ School of Natural and Applied \\ Sciences, Northwest Polytechnical \\ University, Xi'an, Shaanxi 710129, \\ People's Republic of China
}

\begin{abstract}
Let $T$ be the multilinear square operator, respectively with certain smooth kernels and non-smooth kernels defined in (Xue and Yan in J. Math. Anal. Appl. 422:1342-1362, 2015) and (Hormozi et al. in arXiv preprint, 2015), and let $T^{*}$ be its corresponding maximal operator. In this paper, we prove the vector-valued weighted norm boundedness for $T$ and $T^{*}$ and also establish multiple weighted inequalities for their corresponding iterated commutator generated by the vector-valued multilinear operator and BMO function.
\end{abstract}

MSC: Primary 42B20; secondary 42B25; 47G10

Keywords: multilinear square function; vector-valued; weighted estimates; commutators

\section{Introduction}

The importance of the multilinear Littlewood-Paley $g$-function and related multilinear Littlewood-Paley type estimates was shown in PDE and other fields, one can see the works by Coifman et al. [3, 4], David and Journe [5], and also by Fabes et al. [6-8]. Moreover, a class of multilinear square functions was considered in [8], which was used for Kato's problem.

Recently, Xue et al. [9] introduced the multilinear-Paley $g$-function with a convolutiontype kernel in the following way:

$$
g(\vec{f})(x)=\left(\int_{0}^{\infty}\left|\frac{1}{t^{m n}} \int_{\left(\mathbb{R}^{n}\right)^{m}} \psi\left(\frac{y_{1}}{t}, \ldots, \frac{y_{m}}{t}\right) \prod_{j=1}^{m} f_{j}\left(x-y_{j}\right) d \vec{y}\right|^{2} \frac{d t}{t}\right)^{1 / 2}
$$

and obtained the strong $L^{p_{1}}\left(\omega_{1}\right) \times \cdots \times L^{p_{m}}\left(\omega_{m}\right)$ to $L^{p}\left(v_{\vec{\omega}}\right)$ boundedness and the weak type results. Later, Xue and Yan [1] studied a class of multilinear square functions associated with the following more general non-convolution-type kernels.

Definition 1 (Integral smooth condition of C-Z type I) (see [1]) For any $v \in(0, \infty)$, let $K_{v}\left(x, y_{1}, \ldots, y_{m}\right)$ be a locally integrable function defined away from the diagonal $x=y_{1}=$ $\cdots=y_{m}$ in $\left(\mathbb{R}^{n}\right)^{m+1}$ and denote $\vec{y}=\left(y_{1}, \ldots, y_{m}\right)$. We say that $K_{v}$ satisfies the integral condition of $\mathrm{C}-\mathrm{Z}$ type $\mathrm{I}$, if for some positive constants $\gamma, A$, and $B>1$, the following inequalities

(c) $2015 \mathrm{Li}$ and Song. This article is distributed under the terms of the Creative Commons Attribution 4.0 International License (http://creativecommons.org/licenses/by/4.0/), which permits unrestricted use, distribution, and reproduction in any medium, provided you give appropriate credit to the original author(s) and the source, provide a link to the Creative Commons license, and indicate if changes were made. 
hold:

$$
\begin{aligned}
& \left(\int_{0}^{\infty}\left|K_{v}(x, \vec{y})\right|^{2}\right)^{1 / 2} \frac{d v}{v} \leq \frac{A}{\left(\sum_{j=1}^{m}\left|x-y_{j}\right|\right)^{m n}}, \\
& \left(\int_{0}^{\infty}\left|K_{v}(z, \vec{y})-K_{v}(x, \vec{y})\right|^{2} \frac{d v}{v}\right)^{1 / 2} \leq \frac{A|z-x|^{\gamma}}{\left(\sum_{j=1}^{m}\left|x-y_{j}\right|\right)^{m n+\gamma}},
\end{aligned}
$$

whenever $|z-x| \leq \frac{1}{B} \max _{j=1}^{m}\left|x-y_{j}\right|$; and

$$
\left(\int_{0}^{\infty}\left|K_{\nu}(x, \vec{y})-K_{\nu}\left(x, y_{1}, \ldots, y_{i}^{\prime}, \ldots, y_{m}\right)\right|^{2} \frac{d v}{v}\right)^{1 / 2} \leq \frac{A\left|y_{i}-y_{i}^{\prime}\right|^{\gamma}}{\left(\sum_{j=1}^{m}\left|x-y_{j}\right|\right)^{m n+\gamma}}
$$

for any $i \in\{1, \ldots, m\}$, whenever $\left|y_{i}-y_{i}^{\prime}\right| \leq \frac{1}{B}\left|x-y_{i}\right|$.

We define the multilinear square function $T$ by

$$
T(\vec{f})(x)=\left(\int_{0}^{\infty}\left|\int_{\left(\mathbb{R}^{n}\right)^{m}} K_{v}\left(x, y_{1}, \ldots, y_{m}\right) \prod_{j=1}^{m} f_{j}\left(y_{j}\right) d \vec{y}\right|^{2} \frac{d v}{v}\right)^{1 / 2}
$$

for any $\vec{f}=\left(f_{1}, \ldots, f_{m}\right) \in \mathcal{S}\left(\mathbb{R}^{n}\right) \times \cdots \times \mathcal{S}\left(\mathbb{R}^{n}\right)$ and for all $x \notin \bigcap_{j=1}^{m} \operatorname{supp} f_{j}$.

In order to state their results, we first give the definition of multiple weights $A_{\vec{p}}$.

Definition 2 (Multiple weights) (see [10]) Let $1 \leq p_{1}, \ldots, p_{m}<\infty$, and $\frac{1}{p}=\frac{1}{p_{1}}+\cdots+\frac{1}{p_{m}}$. For any $\vec{\omega}=\left(\omega_{1}, \ldots, \omega_{m}\right)$, denote $v_{\vec{\omega}}=\prod_{i=1}^{m} \omega_{i}^{p / p_{i}}$. If

$$
\sup _{B}\left(\frac{1}{|B|} \int_{B} v_{\vec{\omega}}\right)^{1 / p} \prod_{i=1}^{m}\left(\frac{1}{|B|} \int_{B} \omega_{i}^{1-p_{i}^{\prime}}\right)^{1 / p_{i}^{\prime}}<\infty
$$

holds, we say that $\vec{\omega}$ satisfies the $A_{\vec{p}}$ condition. Specially, when $p_{i}=1,\left(\frac{1}{|B|} \int_{B} \omega_{i}^{1-p_{i}^{\prime}}\right)^{1 / p_{i}^{\prime}}$ is understood as $\left(\inf _{B} \omega_{i}\right)^{-1}$.

We will need the easy fact: if each $\omega_{j} \in A_{p_{j}}$, then $\prod_{j=1}^{m} A_{p_{j}} \subset A_{\vec{p}}$. In [10], the multilinear maximal operator $\mathcal{M}$ was defined by

$$
\mathcal{M}(\vec{f})(x)=\sup _{x \in Q} \prod_{i=1}^{m} \frac{1}{|Q|} \int_{Q}\left|f_{i}\left(y_{i}\right)\right| d y_{i},
$$

where the supremum is taken over all cubes $Q$ containing $x$. The easy fact is that $\mathcal{M}(\vec{f})(x) \leq$ $\prod_{i=1}^{m} M f_{i}(x)$, where $M$ is the Hardy-Littlewood maximal operator.

Theorem A (see [1]) Let $T$ be the multilinear square operator defined in (1.4) with the kernel satisfying the integral smooth condition of C-Z type I. Let $1<p_{1}, p_{2}, \ldots, p_{m}<\infty$ and $\frac{1}{p}=\frac{1}{p_{1}}+\cdots+\frac{1}{p_{m}}$. If $\vec{\omega}$ satisfies the $A_{\vec{p}}$ condition, there exists a constant $C$ such that

$$
\|T(\vec{f})\|_{L^{p}\left(v_{\vec{\omega}}\right)} \leq C \prod_{i=1}^{m}\left\|f_{i}\right\|_{L^{p_{i}}\left(\omega_{i}\right)}
$$


Theorem B (see [1]) Let $T$ be the operator defined in (1.4) with the kernel satisfying the integral smooth condition of $C-Z$ type I. Let $0<\delta<1 / m$, the following inequality holds:

$$
M_{\delta}^{\sharp} T(\vec{f})(x) \leq C \mathcal{M}(\vec{f})(x)
$$

for any bounded and compact supported functions $f_{i}, i=1, \ldots, m$.

In order to extend it to a more general case, we recall a class of integral operators $\left\{A_{t}\right\}_{t>0}$ defined in [11], where the operators $A_{t}$ associated with the kernels $a_{t}(x, y)$ are defined by

$$
A_{t} f(x)=\int_{\mathbb{R}^{n}} a_{t}(x, y) f(y) d y
$$

for every function $f \in L^{p}\left(\mathbb{R}^{n}\right), 1 \leq p \leq \infty$, and $a_{t}(x, y)$ satisfies the following size condition:

$$
\left|a_{t}(x, y)\right| \leq h_{t}(x, y):=t^{-n / s} h\left(\frac{|x-y|}{t^{1 / s}}\right) \text { for a fixed constant } s>0
$$

where $h$ is a positive, bounded, decreasing function satisfying

$$
\lim _{r \rightarrow 0} r^{n+\eta} h\left(r^{s}\right)=0
$$

for some $\eta>0$. The above conditions indicate that for some $C>0$ and all $0<\eta \leq \eta^{\prime}$, the kernels $a_{t}(x, y)$ satisfy

$$
\left|a_{t}(x, y)\right| \leq C t^{-n / s}\left(1+t^{-1 / s}|x-y|\right)^{-n-\eta^{\prime}} .
$$

Assumption (H1) Assume that for each $i=1, \ldots, m$, there exist operators $\left\{A_{t}^{(i)}\right\}_{t>0}$ with kernels $a_{t}^{(i)}(x, y)$ satisfying conditions (1.9) and (1.10) with constants $s$ and $\eta$ and that for every $i=1, \ldots, m$, there exist kernels $K_{t, v}^{(i)}$ such that

$$
\begin{aligned}
& \left\langle T\left(f_{1}, \ldots, A_{t}^{(i)} f_{i}, \ldots, f_{m}\right), g\right\rangle \\
& \quad=\int_{\mathbb{R}^{n}}\left(\int_{0}^{\infty}\left|\int_{\left(\mathbb{R}^{n}\right)^{m}} K_{t, v}^{(i)}\left(x, y_{1}, \ldots, y_{m}\right) \prod_{i=1}^{m} f_{i}\left(y_{i}\right) d \vec{y}\right|^{2} \frac{d v}{v}\right)^{1 / 2} g(x) d x
\end{aligned}
$$

for all Schwartz functions $f_{1}, \ldots, f_{m}, g$ with $\bigcap_{k=1}^{m} \operatorname{supp} f_{k} \cap \operatorname{supp} g=\emptyset$.

There exists a function $\phi \in C(\mathbb{R})$ with $\operatorname{supp} \phi \in[-1,1]$ and a constant $\epsilon>0$ so that, for every $i=1, \ldots, m$, we have

$$
\begin{aligned}
& \left(\int_{0}^{\infty}\left|K_{v}(x, \vec{y})-K_{t, v}^{(i)}(x, \vec{y})\right|^{2} \frac{d v}{v}\right)^{1 / 2} \\
& \quad \leq \frac{A}{\left(\sum_{j=1}^{m}\left|x-y_{j}\right|\right)^{m n}} \sum_{k=1, k \neq i}^{m} \phi\left(\frac{\left|y_{i}-y_{k}\right|}{t^{1 / s}}\right)+\frac{A t^{\epsilon / s}}{\left(\sum_{j=1}^{m}\left|x-y_{j}\right|\right)^{m n+\epsilon}},
\end{aligned}
$$

whenever $t^{1 / s} \leq\left|x-y_{i}\right| / 2$. 
Assumption (H2) Assume that there exist operators $\left\{A_{t}\right\}_{t>0}$ with kernels $a_{t}(x, y)$ that satisfy conditions (1.9) and (1.10) with constants $s$ and $\eta$, and there exist kernels $K_{t, v}^{(0)}(x, \vec{y})$ such that

$$
K_{t, v}^{(0)}(x, \vec{y})=\int_{\mathbb{R}^{n}} K_{\nu}(z, \vec{y}) a_{t}(x, z) d z
$$

makes sense for all $(x, \vec{y}) \in\left(\mathbb{R}^{n}\right)^{m+1}$ and $t>0$. Assume also that there exists a function $\phi \in C(\mathbb{R})$ and $\operatorname{supp} \phi \subset[-1,1]$ and a constant $\epsilon>0$ such that

$$
\left(\int_{0}^{\infty}\left|K_{t, v}^{(0)}(x, \vec{y})\right|^{2} \frac{d v}{v}\right)^{1 / 2} \leq \frac{A}{\left(\sum_{k=1}^{m}\left|x-y_{k}\right|\right)^{m n}}
$$

whenever $2 t^{1 / s} \leq \min _{1 \leq j \leq m}\left|x-y_{j}\right|$ and

$$
\begin{aligned}
& \left(\int_{0}^{\infty}\left|K_{v}(x, \vec{y})-K_{t, v}^{(0)}(x, \vec{y})\right|^{2} \frac{d v}{v}\right)^{1 / 2} \\
& \quad \leq \frac{A}{\left(\sum_{j=1}^{m}\left|x-y_{j}\right|\right)^{m n}} \sum_{k=1, k \neq i}^{m} \phi\left(\frac{\left|x-y_{k}\right|}{t^{1 / s}}\right)+\frac{A t^{\epsilon / s}}{\left(\sum_{j=1}^{m}\left|x-y_{j}\right|\right)^{m n+\epsilon}}
\end{aligned}
$$

for some $A>0$, whenever $2 t^{1 / s} \leq \max _{1 \leq j \leq m}\left|x-y_{j}\right|$.

Assumption (H3) Assume that there exist operators $\left\{A_{t}\right\}_{t>0}$ with kernels $a_{t}(x, y)$ that satisfy condition (1.9) and (1.10) with constant $s$ and $\eta$. Also assume that there exist kernels $K_{t, v}^{(0)}$ satisfying (1.13) and positive constants $A$ and $\epsilon$ such that

$$
\left(\int_{0}^{\infty}\left|K_{t, v}^{(0)}(x, \vec{y})-K_{t, v}^{(0)}\left(x^{\prime}, \vec{y}\right)\right|^{2} \frac{d v}{v}\right)^{1 / 2} \leq \frac{A t^{\epsilon / s}}{\left(\sum_{j=1}^{m}\left|x-y_{j}\right|\right)^{m n+\epsilon}}
$$

whenever $2 t^{1 / s} \leq \min _{1 \leq j \leq m}\left|x-y_{j}\right|$ and $2\left|x-x^{\prime}\right| \leq t^{1 / s}$.

We say that the kernels $K_{v}$ generalized the square function kernels if they satisfy (1.1), (1.11), and (1.12) with parameters $m, A, s, \eta, \epsilon$, and we denote their collection by $m-$ $\operatorname{GSFK}(A, s, \eta, \epsilon)$. We say that $T$ is of class $m-\operatorname{GSFO}(A, s, \eta, \epsilon)$ if $T$ has an associated kernel $K_{\nu}$ in $m-\operatorname{GSFK}(A, s, \eta, \epsilon)$.

Theorem C (see [2]) Let $T$ be a multilinear operator in $m-\operatorname{GSFO}(A, s, \eta, \epsilon)$ with a kernel satisfying Assumptions (H2) and (H3). For $1<p_{1}, \ldots, p_{m}<\infty, p \geq 1$ with $\frac{1}{p}=\frac{1}{p_{1}}+\cdots+\frac{1}{p_{m}}$, $\omega \in A_{p}$, the following inequality holds:

$$
\|T(\vec{f})\|_{L^{p}(\omega)} \leq C \prod_{i=1}^{m}\left\|f_{i}\right\|_{L^{p_{i}(\omega)}} .
$$

Theorem $\mathbf{D}$ (see [2]) Let $0<\delta<1 / m$ and $T$ be a multilinear operator in $m-\operatorname{GSFO}(A, s$, $\eta, \epsilon)$ with a kernel satisfying Assumptions (H2) and (H3). Then there exists a constant $C$ such that

$$
M_{\delta}^{\sharp} T(\vec{f})(x) \leq C \prod_{j=1}^{m} M f_{j}(x)
$$

holds for any bounded and compact supported function $f_{i}, i=1,2, \ldots, m$. 
Moreover, the corresponding multilinear maximal square function $T^{*}$ is defined by

$$
T^{*}(\vec{f})(x)=\sup _{\delta>0}\left(\int_{0}^{\infty}\left|\int_{\sum_{i=1}^{m}\left|x-y_{i}\right|^{2}>\delta^{2}} K_{v}(x, \vec{y}) \prod_{k=1}^{m} f_{j}\left(y_{j}\right) d \vec{y}\right|^{2} \frac{d v}{v}\right)^{1 / 2} .
$$

Theorem E (see [2]) Let $T$ be a multilinear operator in $m-\operatorname{GSFO}(A, s, \eta, \epsilon)$ with a kernel satisfying Assumptions (H2) and (H3). For $1<p_{1}, \ldots, p_{m}<\infty, p \geq 1$ with $\frac{1}{p}=\frac{1}{p_{1}}+\cdots+\frac{1}{p_{m}}$, $\omega \in A_{p}$, the following inequality holds:

$$
\left\|T^{*}(\vec{f})\right\|_{L^{p}(\omega)} \leq C \prod_{i=1}^{m}\left\|f_{i}\right\|_{L^{p_{i}(\omega)}}
$$

Theorem $\mathbf{F}$ (see [2]) Let $T$ be a multilinear operator in $m-\operatorname{GSFO}(A, s, \eta, \epsilon)$ with a kernel satisfying Assumptions (H2) and (H3). For any $\eta>0$, there is a constant $C<\infty$ depending on $\eta$ such that the following inequality holds:

$$
T^{*}(\vec{f})(x) \leq C\left(M_{\eta} T(\vec{f})(x)+\prod_{j=1}^{m} M f_{j}(x)\right), \quad \forall x \in \mathbb{R}^{n}
$$

for all $\vec{f}$ in any product of $L^{q_{j}}\left(\mathbb{R}^{n}\right)$ spaces, with $1 \leq q_{j}<\infty$.

\section{Main results}

In this section, we first list some results about vector-valued multilinear operator $T_{q}$ and the corresponding vector-valued maximal multilinear operator $T_{q}^{*}$ which are defined, respectively, by

$$
\begin{aligned}
& T_{q}(\vec{f})(x)=\|T(\vec{f})(x)\|_{\ell q}=\left(\sum_{k=1}^{\infty}\left|T\left(f_{1 k}, \ldots, f_{m k}\right)(x)\right|^{q}\right)^{1 / q}, \\
& T_{q}^{*}(\vec{f})(x)=\left\|T^{*}(\vec{f})(x)\right\|_{\ell q}=\left(\sum_{k=1}^{\infty}\left|T^{*}\left(f_{1 k}, \ldots, f_{m k}\right)(x)\right|^{q}\right)^{1 / q},
\end{aligned}
$$

where $\vec{f}=\left(f_{1}, \ldots, f_{m}\right)$ with $f_{i}=\left\{f_{i k}\right\}_{k=1}^{\infty}$.

Theorem 3 Assume that $T$ is a multilinear square operator defined in (1.4) with the kernel satisfying the integral condition of C-Z type I. Let $1<p_{1}, p_{2}, \ldots, p_{m}<\infty, 1<q_{1}, q_{2}, \ldots, q_{m}<$ $\infty$, and $1 / m<p, q<\infty$ with $\frac{1}{p}=\frac{1}{p_{1}}+\cdots+\frac{1}{p_{m}}, \frac{1}{q}=\frac{1}{q_{1}}+\cdots+\frac{1}{q_{m}}$. If $\left(\omega_{1}^{p_{1}}, \ldots, \omega_{m}^{p_{m}}\right) \in$ $\left(A_{p_{1}}, \ldots, A_{p_{m}}\right)$, the following inequality holds:

$$
\left\|T_{q}(\vec{f})\right\|_{L^{p}\left(\prod_{j=1}^{m} \omega_{j}^{p}\right)} \leq C \prod_{j=1}^{m}\|\| f_{j}\left\|_{\ell^{q_{j}}}\right\|_{L^{p_{j}}\left(\omega_{j}^{p_{j}}\right)}
$$

Theorem 4 Assume that $T$ is a multilinear square operator defined in (1.4) with the kernel satisfying the integral condition of C-Z type I. Let $1 \leq p_{1}, p_{2}, \ldots, p_{m}<\infty, 1<q_{1}, q_{2}, \ldots, q_{m}<$ $\infty$, and $0<p, q<\infty$ with $\frac{1}{p}=\frac{1}{p_{1}}+\cdots+\frac{1}{p_{m}}, \frac{1}{q}=\frac{1}{q_{1}}+\cdots+\frac{1}{q_{m}}$. 
(i) If $1 \leq p_{1}, p_{2}, \ldots, p_{m}<\infty$ and $\omega \in A_{p_{1}} \cap \cdots \cap A_{p_{m}}$, there exists a constant $C>0$ such that

$$
\left\|T_{q}(\vec{f})\right\|_{L^{p}(\omega)} \leq C \prod_{j=1}^{m}\|\| f_{j}\left\|_{\ell^{q_{j}}}\right\|_{L^{p_{j}(\omega)}} .
$$

(ii) If at least one $p_{j}=1$ and $\omega \in A_{1}$, there exists a constant $C>0$ such that

$$
\left\|T_{q}(\vec{f})\right\|_{L^{p, \infty}(\omega)} \leq C \prod_{j=1}^{m}\|\| f_{j}\left\|_{\ell^{q_{j}}}\right\|_{L^{p_{j}(\omega)}} .
$$

Next, we show the results for the multilinear square operator $T$ with non-smooth kernels and its corresponding maximal operator $T^{*}$. Meanwhile, we also establish multiple weighted inequalities for their corresponding iterated commutator generated by the vector-valued multilinear operator and BMO function. We will state our results as follows.

Theorem 5 Let $T$ be a multilinear operator in $m-\operatorname{GSFO}(A, s, \eta, \epsilon)$ with a kernel satisfying Assumptions (H2) and (H3). Let $1<p_{1}, p_{2}, \ldots, p_{m}<\infty, 1<q_{1}, q_{2}, \ldots, q_{m}<\infty$ and $1 / m<$ $p, q<\infty$ with $\frac{1}{p}=\frac{1}{p_{1}}+\cdots+\frac{1}{p_{m}}, \frac{1}{q}=\frac{1}{q_{1}}+\cdots+\frac{1}{q_{m}}$. If $\left(\omega_{1}^{p_{1}}, \ldots, \omega_{m}^{p_{m}}\right) \in\left(A_{p_{1}}, \ldots, A_{p_{m}}\right)$, there exists a constant $C>0$ such that

$$
\left\|T_{q}(\vec{f})\right\|_{L^{p}\left(\prod_{j=1}^{m} \omega_{j}^{p}\right)} \leq C \prod_{j=1}^{m}\|\| f_{j}\left\|_{\ell^{q_{j}}}\right\|_{L^{p_{j}}\left(\omega_{j}^{p_{j}}\right)} .
$$

A similar estimate also holds true for the corresponding maximal operator $T^{*}$.

Theorem 6 Let $T$ be a multilinear operator in $m-\operatorname{GSFO}(A, s, \eta, \epsilon)$ with a kernel satisfying Assumptions (H2) and (H3). Let $1 \leq p_{1}, p_{2}, \ldots, p_{m}<\infty, 1<q_{1}, q_{2}, \ldots, q_{m}<\infty$ and $0<p, q<$ $\infty$ with $\frac{1}{p}=\frac{1}{p_{1}}+\cdots+\frac{1}{p_{m}}, \frac{1}{q}=\frac{1}{q_{1}}+\cdots+\frac{1}{q_{m}}$.

(i) If $1 \leq p_{1}, p_{2}, \ldots, p_{m}<\infty$ and $\omega \in A_{p_{1}} \cap \cdots \cap A_{p_{m}}$, the following inequality holds:

$$
\left\|T_{q}(\vec{f})\right\|_{L^{p}(\omega)} \leq C \prod_{j=1}^{m}\|\| f_{j}\left\|_{\ell^{q_{j}}}\right\|_{L^{p_{j}(\omega)}} .
$$

(ii) If at least one $p_{j}=1$ and $\omega \in A_{1}$, the following inequality holds:

$$
\left\|T_{q}(\vec{f})\right\|_{L^{p, \infty}(\omega)} \leq C \prod_{j=1}^{m}\|\| f_{j}\left\|_{\ell^{q_{j}}}\right\|_{L^{p_{j}(\omega)}} .
$$

Similar estimates also hold true for the corresponding maximal operators $T^{*}$.

The commutator associated with $T$ is given by

$$
\begin{aligned}
T_{\Pi \vec{b}}(\vec{f})(x) & =\left[b_{1},\left[b_{2}, \ldots\left[b_{\ell-1},\left[b_{\ell}, T\right]_{\ell}\right]_{\ell-1} \ldots\right]_{2}\right]_{1}(\vec{f})(x) \\
& =\int_{\left(\mathbb{R}^{n}\right)^{m}} \prod_{j=1}^{\ell}\left(b_{j}(x)-b_{j}(y)\right) K\left(x, y_{1}, \ldots, y_{m}\right) \prod_{i=1}^{m} f_{i}\left(y_{i}\right) d \vec{y}
\end{aligned}
$$

where $1 \leq \ell \leq m$. 
For simplicity of notation, for the sequence $\left\{\vec{f}_{k}\right\}_{k=1}^{\infty}=\left\{f_{1 k}, \ldots, f_{m k}\right\}_{k=1}^{\infty}$ of vector functions, the commutator associated with a vector-valued $T_{q}$ can be defined by

$$
T_{\Pi \vec{b}, q}(\vec{f})(x)=\left\|T_{\Pi \vec{b}}(\vec{f})(x)\right\|_{\ell q}=\left(\sum_{k=1}^{\infty}\left|T_{\Pi \vec{b}}\left(\vec{f}_{k}\right)(x)\right|^{q}\right)^{1 / q} .
$$

Theorem 7 Assume that $T$ is a multilinear operator in $m-\operatorname{GSFO}(A, s, \eta, \epsilon)$ with kernel satisfying Assumptions (H2) and (H3). Let $1 / m<p<\infty, \frac{1}{p}=\frac{1}{p_{1}}+\cdots+\frac{1}{p_{m}}$ with $1<p_{1}, \ldots, p_{m}<$ $\infty, 1 / m<q<\infty$, and $\frac{1}{q}=\frac{1}{q_{1}}+\cdots+\frac{1}{q_{m}}$ with $1<q_{1}, \ldots, q_{m}<\infty$. Suppose that $\vec{\omega} \in A_{\vec{p}}$ and $\vec{b} \in(B M O)^{\ell}$.

(i) There exists a constant $C>0$ such that

$$
\left\|T_{\Pi \vec{b}, q}(\vec{f})\right\|_{L^{p}\left(v_{\omega}\right)} \leq \prod_{j=1}^{\ell}\left\|b_{j}\right\|_{B M O} \prod_{j=1}^{m}\|\| f\left\|_{q_{j}}\right\|_{L^{p_{j}}\left(M \omega_{j}\right)} .
$$

(ii) If $\omega_{j} \in A_{p_{j}}$, there exists a constant $C>0$ such that

$$
\left\|T_{\Pi \vec{b}, q}(\vec{f})\right\|_{L^{p}\left(v_{\omega}\right)} \leq \prod_{j=1}^{\ell}\left\|b_{j}\right\|_{B M O} \prod_{j=1}^{m}\|\| f\left\|_{q_{j}}\right\|_{L^{p_{j}\left(\omega_{j}\right)}} .
$$

\section{The proof of Theorem 3}

Since $\omega_{j} \in A_{p_{j}}$, by the previous statement, $\prod_{j=1}^{m} \omega_{j}^{p_{j}} \in A_{\vec{p}}$. Writing $v_{\vec{\omega}}=\prod_{j=1}^{m}\left(\omega_{j}^{p_{j}}\right)^{p / p_{j}}=$ $\prod_{j=1}^{m} \omega_{j}^{p}$, Theorem A implies that

$$
\|T(f)\|_{L^{p}\left(\prod_{j=1}^{m} \omega_{j}^{p}\right)} \leq C \prod_{j=1}^{m}\left\|f_{j}\right\|_{L^{p_{j}}}\left(\omega_{j}^{p_{j}}\right) .
$$

We will apply the following lemma to get the desirable result.

Lemma 8 (see [12]) Let $\mathcal{T}$ be an m-linear operator, and let $1<s_{1}, \ldots, s_{m}<\infty$ and $1 / m<s<$ $\infty$ be fixed indices such that $\frac{1}{s}=\frac{1}{s_{1}}+\cdots+\frac{1}{s_{m}}$. For $\left(\omega_{1}^{s_{1}}, \ldots, \omega_{m}^{s_{m}}\right) \in\left(A_{s_{1}}, \ldots, A_{s_{m}}\right)$, the following estimate holds:

$$
\|\mathcal{T}(\vec{f})\|_{L^{s}\left(\prod_{j=1}^{m} \omega_{j}^{s}\right)} \prod_{j=1}^{m}\left\|f_{j}\right\|_{L^{s_{j}}}\left(\omega_{j}^{s_{j}}\right) .
$$

Then, for all indices, $1<p_{1}, \ldots, p_{m}<\infty$ and $1 / m<p<\infty$ satisfy $\frac{1}{p}=\frac{1}{p_{1}}+\cdots+\frac{1}{p_{m}}$, $1<q_{1}, \ldots, q_{m}<\infty$, and $1 / m<q<\infty$ such that $\frac{1}{q}=\frac{1}{q_{1}}+\cdots+\frac{1}{q_{m}}$, and all $\left(\omega_{1}^{p_{1}}, \ldots, \omega_{m}^{p_{m}}\right) \in$ $\left(A_{p_{1}}, \ldots, A_{p_{m}}\right)$. Then the following inequality holds:

$$
\left\|\mathcal{T}_{q}(\vec{f})\right\|_{L^{p}\left(\prod_{j=1}^{m} \omega_{j}^{p}\right)} \leq C \prod_{j=1}^{m}\|\| f_{j}\left\|_{\ell^{q_{j}}}\right\|_{L^{p_{j}}\left(\omega_{j}^{p_{j}}\right)} .
$$

\section{The proof of Theorem 5}

We first state the following Fefferman-Stein inequality. 
Lemma 9 (see [13]) Let $0<p, \delta<\infty$ and $\omega$ be any Mockenhaupt $A_{\infty}$ weight. Then there exists a constant $C$ independent off such that the inequality

$$
\int_{\mathbb{R}^{n}}\left(M_{\delta} f(x)\right)^{p} \omega(x) d x \leq C \int_{\mathbb{R}^{n}}\left(M_{\delta}^{\sharp} f(x)\right)^{p} \omega(x) d x,
$$

holds for any function $f$ for which the left-hand side is finite.

Lemma 10 (see [12]) For $\left(\omega_{1}, \ldots, \omega_{m}\right) \in\left(A_{p_{1}}, \ldots, A_{p_{m}}\right)$ with $1 \leq p_{1}, \ldots, p_{m}<\infty$ and for $0<$ $\theta_{1}, \ldots, \theta_{m}<1$ such that $\theta_{1}+\cdots+\theta_{m}=1$, we have $\omega_{1}^{\theta_{1}} \cdots \omega_{1}^{\theta_{1}} \in A_{\max \left\{p_{1}, \ldots, p_{m}\right\}}$.

Note that $\left(\omega_{1}^{p_{1}}, \ldots, \omega_{m}^{p_{m}}\right) \in\left(A_{p_{1}}, \ldots, A_{p_{m}}\right)$, and Lemma 10 indicates that $\prod_{j=1}^{m} \omega_{j}^{p}=$ $\prod_{j=1}^{m}\left(\omega_{j}^{p_{j}}\right)^{p / p_{j}} \in A_{\max \left\{p_{1}, \ldots, p_{m}\right\}} \subset A_{\infty}$.

Exploiting Lemma 3.3 in [2] and the standard argument, we obtain $\left\|M_{\delta} T(\vec{f})\right\|_{L^{p}\left(\prod_{j=1}^{m} \omega_{j}^{p}\right)}<$ $\infty$. Together with Theorem $\mathrm{D}$, we have

$$
\begin{aligned}
\|T(\vec{f})\|_{L^{p}\left(\prod_{j=1}^{m} \omega_{j}^{p}\right)} & \leq\left\|M_{\delta} T(\vec{f})\right\|_{L^{p}\left(\prod_{j=1}^{m} \omega_{j}^{p}\right)} \\
& \leq C\left\|M_{\delta}^{\sharp} T(\vec{f})\right\|_{L^{p}\left(\prod_{j=1}^{m} \omega_{j}^{p}\right)} \\
& \leq C\left\|\prod_{j=1}^{m} M f_{j}\right\|_{L^{p}\left(\prod_{j=1}^{m} \omega_{j}^{p}\right)} \\
& \leq C \prod_{j=1}^{m}\left\|M f_{j}\right\|_{L^{p_{j}}\left(\omega_{j}^{p_{j}}\right)} \\
& \leq C \prod_{j=1}^{m}\left\|f_{j}\right\|_{L^{p_{j}}\left(\omega_{j}^{p_{j}}\right)^{p_{j}}}
\end{aligned}
$$

By Lemma 8, we finish the proof of Theorem 5 .

The estimate for $T^{*}$ will follow from Lemma 8 , Theorem $\mathrm{F}$, and the following argument:

$$
\begin{aligned}
\left\|T^{*}(\vec{f})\right\|_{L^{p}\left(\prod_{j=1}^{m} \omega_{j}^{p}\right)} & \leq C\left(\left\|M_{\eta} T(\vec{f})\right\|_{L^{p}\left(\prod_{j=1}^{m} \omega_{j}^{p}\right)}+\left\|\prod_{j=1}^{m} M f_{j}\right\|_{L^{p}\left(\prod_{j=1}^{m} \omega_{j}^{p}\right)}\right) \\
& \leq C\left(\left\|M|T(\vec{f})|^{\eta}\right\|_{L^{\frac{p}{\eta}}\left(\prod_{j=1}^{m} \omega_{j}^{p}\right)}^{\frac{1}{\eta}}+\left\|\prod_{j=1}^{m} M f_{j}\right\|_{L^{p}\left(\prod_{j=1}^{m} \omega_{j}^{p}\right)}\right) \\
& \leq C\left(\left\|\left.T(\vec{f})\right|^{\eta}\right\|_{L^{\frac{p}{\eta}}\left(\prod_{j=1}^{m} \omega_{j}^{p}\right)}^{\frac{1}{\eta}}+\left\|\prod_{j=1}^{m} M f_{j}\right\|_{L^{p}\left(\prod_{j=1}^{m} \omega_{j}^{p}\right)}\right) \\
& \leq C\left(\|T(\vec{f})\|_{L^{p}\left(\prod_{j=1}^{m} \omega_{j}^{p}\right)}+\left\|\prod_{j=1}^{m} M f_{j}\right\|_{L^{p}\left(\prod_{j=1}^{m} \omega_{j}^{p}\right)}\right) \\
& \leq C\left\|\prod_{j=1}^{m} M f_{j}\right\|_{L^{p}\left(\prod_{j=1}^{m} \omega_{j}^{p}\right)} .
\end{aligned}
$$

\section{The proofs of Theorem 4 and Theorem 6}

In order to prove these theorems, first we introduce the following lemmas. 
Let $\mathcal{F}$ denote a family of ordered pairs of non-negative, measurable functions $(f, g)$, if we say that for some $p, 0<p<\infty$, and $\omega \in A_{\infty}$,

$$
\int_{\mathbb{R}^{n}} f(x)^{p} \omega(x) d x \leq C \int_{\mathbb{R}^{n}} g(x)^{p} \omega(x) d x
$$

and we denote it by $(f, g) \in \mathcal{F}$.

Lemma 11 (see [14]) Given a family $\mathcal{F}$, suppose that for some $p_{0}, 0<p_{0}<\infty$, and for every weight $\omega \in A_{\infty},(f, g) \in \mathcal{F}$. Then we have, for all $0<p, q<\infty$ and $\omega \in A_{\infty}$,

$$
\left\|\left(\sum_{k}\left(f_{k}\right)^{q}\right)^{1 / q}\right\|_{L^{p}(\omega)} \leq C\left\|\left(\sum_{k}\left(g_{k}\right)^{q}\right)^{1 / q}\right\|_{L^{p}(\omega)}, \quad\left\{\left(f_{k}, g_{k}\right)\right\}_{k} \subset \mathcal{F} .
$$

For all $0<p, q<\infty, 0<s \leq \infty$, and $\omega \in A_{\infty}$

$$
\left\|\left(\sum_{k}\left(f_{k}\right)^{q}\right)^{1 / q}\right\|_{L^{p, s}(\omega)} \leq C\left\|\left(\sum_{k}\left(g_{k}\right)^{q}\right)^{1 / q}\right\|_{L^{p, s}(\omega)}, \quad\left\{\left(f_{k}, g_{k}\right)\right\}_{k} \subset \mathcal{F} .
$$

Lemma 12 (see [15])

(i) Let $1<q<\infty$ and $1 \leq p<\infty$, there is a constant $C_{r, p}$ such that

$$
\left\|\left(\sum_{k}\left|M f_{k}(x)\right|^{q}\right)^{1 / q}\right\|_{L^{p, \infty}(\omega)} \leq C_{q, p}\left\|\left(\sum_{k}\left|f_{k}(x)\right|^{q}\right)^{1 / q}\right\|_{L^{p}(\omega)}
$$

if and only if $\omega \in A_{p}$.

(ii) Let $1<q<\infty$ and $1<p<\infty$, there is a constant $C_{q, p}$ such that

$$
\left\|\left(\sum_{k}\left|M f_{k}(x)\right|^{q}\right)^{1 / q}\right\|_{L^{p}(\omega)} \leq C_{q, p}\left\|\left(\sum_{k}\left|f_{k}(x)\right|^{q}\right)^{1 / q}\right\|_{L^{p}(\omega)}
$$

if and only if $\omega \in A_{p}$.

By Theorem B, Theorem D, and Theorem F, together with the argument from Section 3 and (4.2), we have

$$
\|\mathcal{T}(\vec{f})\|_{L^{p}(\omega)} \leq C\left\|\prod_{j=1}^{m} M f_{j}\right\|_{L^{p}(\omega)} .
$$

Here $\mathcal{T}$ can be replaced by $T$ and $T^{*}$ which are from Theorem 4 and Theorem 6 .

We apply Lemma 11 to $\left(T(\vec{f}), \prod_{j=1}^{m} M f_{j}\right) \in \mathcal{F}$, and by Lemma 12 we get the desirable results.

\section{The proof of Theorem 7}

In order to prove Theorem 7, first we will list some notations and lemmas:

$$
\mathcal{M}\left(\|\vec{f}\|_{\ell^{q}}\right)(x):=\sup _{x \in Q} \prod_{j=1}^{m} \frac{1}{|Q|} \int_{Q}\left\|f_{j}\left(y_{j}\right)\right\|_{\ell^{q_{j}}} d y_{j},
$$




$$
\begin{aligned}
& \mathcal{M}_{L(\log L)}\left(\|\vec{f}\|_{\ell q}\right)(x):=\sup _{x \in Q} \prod_{j=1}^{m}\|\| f_{j}\left\|_{\ell^{q_{j}}}\right\|_{L(\log L), Q^{\prime}} \\
& \mathcal{M}_{\rho}\left(\|\vec{f}\|_{\ell q}\right)(x) \\
& \quad:=\sup _{x \in Q} \sum_{\nu=0}^{\infty} 2^{-v n \ell} \prod_{j \in \rho} \frac{1}{|Q|} \int_{Q}\left\|f_{j}\left(y_{j}\right)\right\|_{\ell^{q_{j}}} d y_{j} \prod_{j \in \rho^{\prime}} \frac{1}{\left|2^{v} Q\right|} \int_{2^{v} Q}\left\|f_{j}\left(y_{j}\right)\right\|_{\ell^{q_{j}}} d y_{j},
\end{aligned}
$$

where $\rho=\left\{j_{1}, \ldots, j_{\ell}\right\} \subset\{1, \ldots, m\}, 1 \leq \ell<m$ and $\rho^{\prime}=\{1, \ldots, m\} \backslash \rho$.

Lemma 13 (see [10]) Let $1<p_{1}, \ldots, p_{m}<\infty, \frac{1}{p}=\frac{1}{p_{1}}+\cdots+\frac{1}{p_{m}}, \vec{P}=\left(p_{1}, \ldots, p_{m}\right), \vec{\omega} \in A_{\vec{P}}$, and $\rho=\left\{j_{1}, \ldots, j_{\ell}\right\} \subset\{1, \ldots, m\}, 1 \leq \ell<m$. Then $\mathcal{M}, \mathcal{M}_{L(\log L)}, \mathcal{M}_{\rho}$ are bounded from $L^{p_{1}}\left(\omega_{1}\right) \times$ $\cdots \times L^{p_{m}}\left(\omega_{m}\right)$ to $L^{p}\left(v_{\omega}\right)$.

Lemma 14 Let $T$ be a multilinear operator in $m-\operatorname{GSFO}(A, s, \eta, \epsilon)$ with kernel satisfying Assumptions (H2) and (H3). Assume that $1 \leq \ell<m, \rho=\left\{j_{1}, \ldots, j_{\ell}\right\}$, and $1 / m<q<\infty, 1 \leq$ $q_{1}, \ldots, q_{m}<\infty$ with $\frac{1}{q}=\frac{1}{q_{1}}+\cdots+\frac{1}{q_{m}}$. Then there exists a constant $C>0$ such that

$$
M_{\delta}^{\sharp} T_{q}(\vec{f})(x) \leq C\left(\mathcal{M}\left(\|\vec{f}\|_{\ell q}\right)(x)+\mathcal{M}_{\rho}\left(\|\vec{f}\|_{\ell q}\right)(x)\right) .
$$

Proof For a point $x$ and a cube $Q \ni x$, to obtain (6.4), it suffices to prove for $0<\delta<1 / m$,

$$
\left(\frac{1}{|Q|} \int_{Q}\|T(\vec{f})(z)-c\|_{\ell q}^{\delta} d z\right)^{1 / \delta} \leq C\left(\mathcal{M}\left(\|\vec{f}\|_{\ell q}\right)(x)+\mathcal{M}_{\rho}\left(\|\vec{f}\|_{\ell q}\right)(x)\right)
$$

for some constant $c$ to be determined later.

Write $\vec{f}_{k}=\vec{f}_{k}^{0}+\vec{f}_{k}^{\infty}$, where $\left\{\vec{f}_{k}^{0}\right\}_{k=1}^{\infty}=\left\{\vec{f}_{k}^{0} \chi_{Q^{*}}\right\}_{k=1}^{\infty}=\left\{f_{1 k} \chi_{Q^{*}}, \ldots, f_{m k} \chi_{Q^{*}}\right\}$ and $Q^{*}=(8 \sqrt{n}+4) Q$. Let $c=\sum_{\alpha_{1}, \ldots, \alpha_{m}} T\left(\vec{f}^{\alpha}\right)(x)$ and in the sum each $\alpha_{j}=0$ or $\infty$ and in each term there is at least one $\alpha_{j}=\infty$. Then

$$
\begin{aligned}
& \left(\frac{1}{|Q|} \int_{Q}\|T(\vec{f})(z)-c\|_{\ell q}^{\delta} d z\right)^{1 / \delta} \\
& \leq C\left(\frac{1}{|Q|} \int_{Q}\left|T_{q}\left(\vec{f}^{0}\right)(z)\right|^{\delta} d z\right)^{1 / \delta} \\
& \quad+C \sum_{\alpha_{1}, \ldots, \alpha_{m}}\left(\frac{1}{|Q|} \int_{Q}\left\|T\left(\vec{f}^{\alpha}\right)(z)-T\left(\vec{f}^{\alpha}\right)(x)\right\|_{\ell^{q}}^{\delta} d z\right)^{1 / \delta} \\
& :=I+\sum_{\alpha_{1}, \ldots, \alpha_{m}} I I_{\alpha_{1}, \ldots, \alpha_{m}},
\end{aligned}
$$

where in each term of the last sum there is at least one $\alpha_{j}=\infty$.

Kolmogorov's inequality and Theorem 6 implies that

$$
\begin{aligned}
I & \leq C\left\|T_{q}\left(\vec{f}^{0}\right)\right\|_{L^{1 / m, \infty}\left(Q, \frac{d z}{Q Q}\right)} \\
& \leq C \prod_{j=1}^{m} \frac{1}{|Q|} \int_{Q}\left\|f_{j}(z)\right\|_{\ell^{q_{j}}} d z \\
& \leq C \mathcal{M}\left(\|\vec{f}\|_{\ell q}\right) .
\end{aligned}
$$


We proceed to the estimate for $I I_{\alpha_{1}, \ldots, \alpha_{m}}$. Here we choose $t=[2 \sqrt{n} \ell(Q)]^{s}$. If $\alpha_{1}=\cdots \alpha_{m}=$ $\infty$, we have

$$
\begin{aligned}
I I_{\infty, \ldots, \infty} & \leq \frac{C}{|Q|} \int_{Q}\left\|T\left(\vec{f}^{\alpha}\right)(z)-T\left(\vec{f}^{\alpha}\right)(x)\right\|_{\ell q} d z \\
& \leq \frac{C}{|Q|} \int_{Q}\left(\sum_{k=1}^{\infty}\left|T\left(\vec{f}_{k}^{\infty}\right)(z)-T\left(\vec{f}_{k}^{\infty}\right)(x)\right|^{q}\right)^{1 / q} d z \\
& \leq \frac{C}{|Q|} \int_{Q}\left(\left.\left.\sum_{k=1}^{\infty}\left|\int_{0}^{\infty}\right| \int_{\left(\mathbb{R}^{n} \backslash Q^{*}\right)^{m}}\left(K_{\nu}(z, \vec{y})-K_{v}(x, \vec{y})\right) \prod_{j=1}^{m} f_{j k}\left(y_{j}\right) d \vec{y}\right|^{2} \frac{d \nu}{v}\right|^{q / 2}\right)^{1 / q} d z,
\end{aligned}
$$

applying Minkowski's inequality, we get

$$
\begin{aligned}
\frac{C}{|Q|} \int_{Q}\left(\left.\left.\sum_{k=1}^{\infty}\left|\int_{0}^{\infty}\right| \int_{\left(\mathbb{R}^{n} \backslash Q^{*}\right)^{m}}\left(K_{v}(z, \vec{y})-K_{v}(x, \vec{y})\right) \prod_{j=1}^{m} f_{j k}\left(y_{j}\right) d \vec{y}\right|^{2} \frac{d v}{v}\right|^{q / 2}\right)^{1 / q} d z \\
\leq \frac{C}{|Q|} \int_{Q}\left(\sum_{k=1}^{\infty}\left|\int_{\left(\mathbb{R}^{n} \backslash Q^{*}\right)^{m}}\left(\int_{0}^{\infty}\left|K_{v}(z, \vec{y})-K_{v}(x, \vec{y})\right|^{2} \frac{d v}{v}\right)^{1 / 2} \prod_{j=1}^{m} f_{j k}\left(y_{j}\right) d \vec{y}\right|^{q}\right)^{1 / q} d z \\
\leq \frac{C}{|Q|} \int_{Q}\left(\sum_{k=1}^{\infty}\left|\int_{\left(\mathbb{R}^{n} \backslash Q^{*}\right)^{m}}\left(\int_{0}^{\infty}\left|K_{v}(z, \vec{y})-K_{t, v}^{(0)}(z, \vec{y})\right|^{2} \frac{d v}{v}\right)^{1 / 2} \prod_{j=1}^{m} f_{j k}\left(y_{j}\right) d \vec{y}\right|^{q}\right)^{1 / q} d z \\
\quad+\frac{C}{|Q|} \int_{Q}\left(\sum_{k=1}^{\infty} \mid \int_{\left(\mathbb{R}^{n} \backslash Q^{*}\right)^{m}}\left(\int_{0}^{\infty}\left|K_{t, v}^{(0)}(z, \vec{y})-K_{t, v}^{(0)}(x, \vec{y})\right|^{2} \frac{d v}{v}\right)^{1 / 2}\right. \\
\left.\quad \times \prod_{j=1}^{m} f_{j k}\left(y_{j}\right) d \vec{y} \mid\right)^{q} d z \\
\quad+\frac{C}{|Q|} \int_{Q}\left(\sum_{k=1}^{\infty}\left|\int_{\left(\mathbb{R}^{n} \backslash Q^{*}\right)^{m}}\left(\int_{0}^{\infty}\left|K_{t, v}^{(0)}(x, \vec{y})-K_{v}(x, \vec{y})\right|^{2} \frac{d v}{v}\right)^{1 / 2} \prod_{j=1}^{m} f_{j k}\left(y_{j}\right) d \vec{y}\right|^{q}\right)^{1 / q} d z \\
:=I I_{\infty, \ldots, \ldots}^{1}+I I_{\infty, \ldots, \infty}^{2}+I I_{\infty, \ldots, \infty}^{3}
\end{aligned}
$$

Because of $z \in Q$ and $y_{j} \in \mathbb{R}^{n} \backslash(8 \sqrt{n}+4) Q$, we obtain $\left|y_{j}-z\right|>(4 \sqrt{n}+1) \ell(Q)>2 t^{1 / s}$ for all $j=1, \ldots, m$. Assumption $(\mathrm{H} 2)$ gives

$$
\begin{aligned}
I I_{\infty, \ldots, \infty}^{1} & \leq \frac{C}{|Q|} \int_{Q} \int_{\left(\mathbb{R}^{n} \backslash Q^{*}\right)^{m}} \frac{A t^{\epsilon / s}}{\left(\sum_{j=1}^{m}\left|z-y_{j}\right|\right)^{m n+\epsilon}} \prod_{j=1}^{m}\left\|f_{j}\left(y_{j}\right)\right\|_{\ell^{q_{j}}} d \vec{y} d z \\
& \leq \sum_{k=1}^{\infty} \frac{1}{2^{k \epsilon}} \prod_{j=1}^{m} \frac{1}{2^{(k+1) n}\left|Q^{*}\right|} \int_{2^{k+1} Q^{*}}\left\|f_{j}\left(y_{j}\right)\right\|_{\ell^{q_{j}}} d y_{j} \\
& \leq C \mathcal{M}\left(\|\vec{f}\|_{\ell q}\right)(x) .
\end{aligned}
$$

Since $x, z \in Q,|z-x| \leq \sqrt{n} \ell(Q) \leq 1 / 2 t^{1 / s}$. Noting that $\left|y_{j}-z\right|>(4 \sqrt{n}+1) \ell(Q)>2 t^{1 / s}$ for all $j=1, \ldots, m$, applying Assumption (H3) and a similar argument to $I I^{1}$, we have $I I_{\infty, \ldots, \infty}^{2} \leq$ $C \mathcal{M}\left(\|\vec{f}\|_{\ell^{q}}\right)(x)$. Similarly, we also get $I_{\infty, \ldots, \infty}^{3} \leq C \mathcal{M}\left(\|\vec{f}\|_{\ell^{q}}\right)(x)$. 
Now let us consider the typical case of $I I_{\alpha_{1}, \ldots, \alpha_{m}}$, that is, $\alpha_{1}=\cdots=\alpha_{h}=\infty$ and $\alpha_{h+1}=\cdots=$ $\alpha_{m}=0,1 \leq h<m$,

$$
\begin{aligned}
I I_{\infty, \ldots, 0} \leq & \frac{C}{|Q|} \int_{Q}\left(\sum_{k=1}^{\infty} \mid \int_{\left(\mathbb{R}^{n}\right)^{m}}\left(\int_{0}^{\infty}\left|K_{v}(z, \vec{y})-K_{t, v}^{(0)}(z, \vec{y})\right|^{2} \frac{d v}{v}\right)^{1 / 2}\right. \\
& \left.\times\left.\prod_{j=1}^{h} f_{j k}^{\infty} \prod_{j=h+1}^{m} f_{j k}^{0} d \vec{y}\right|^{q}\right)^{1 / q} d z \\
& +\frac{C}{|Q|} \int_{Q}\left(\sum_{k=1}^{\infty} \mid \int_{\left(\mathbb{R}^{n}\right)^{m}}\left(\int_{0}^{\infty}\left|K_{t, v}^{(0)}(z, \vec{y})-K_{t, v}^{(0)}(x, \vec{y})\right|^{2} \frac{d v}{v}\right)^{1 / 2}\right. \\
& \left.\times\left.\prod_{j=1}^{h} f_{j k}^{\infty} \prod_{j=h+1}^{m} f_{j k}^{0} d \vec{y}\right|^{q}\right)^{1 / q} d z \\
& +\frac{C}{|Q|} \int_{Q}\left(\sum_{k=1}^{\infty} \mid \int_{\left(\mathbb{R}^{n}\right)^{m}}\left(\int_{0}^{\infty}\left|K_{t, v}^{(0)}(x, \vec{y})-K_{v}(x, \vec{y})\right|^{2} \frac{d v}{v}\right)^{1 / 2}\right. \\
& \left.\times\left.\prod_{j=1}^{h} f_{j k}^{\infty} \prod_{j=h+1}^{m} f_{j k}^{0} d \vec{y}\right|^{q}\right)^{1 / q} d z \\
:= & I I_{\infty, \ldots, 0}^{1}+I I_{\infty, \ldots, 0}^{2}+I I_{\infty, \ldots, 0}^{3} .
\end{aligned}
$$

For $I I_{\infty, \ldots, 0}^{1}$, by Assumption (H2), we have

$$
\begin{aligned}
I I_{\infty, \ldots, 0,}^{1} \leq & \frac{C}{|Q|} \int_{Q}\left(\int_{\left(\mathbb{R}^{n} \backslash Q^{*}\right)^{h}} \frac{A t^{\epsilon / s} \prod_{j=1}^{h}\left\|f_{j}\right\|_{\ell^{q_{j}}} d y_{j}}{\left(\sum_{j \in\{1, \ldots, h\}}^{m}\left|z-y_{j}\right|\right)^{m n+\epsilon}}\right. \\
& \left.+\int_{\left(\mathbb{R}^{n} \backslash Q^{*}\right)^{h}} \frac{A \prod_{j=1}^{h}\left\|f_{j}\right\|_{\ell^{q_{j}}} d y_{j}}{\left(\sum_{j \in\{1, \ldots, h\}}^{m}\left|z-y_{j}\right|\right)^{m n}}\right) \prod_{j=h+1}^{m} \int_{Q^{*}}\left\|f_{j}\right\|_{\ell^{q_{j}}} d y_{j} d z \\
\leq & \left(\sum_{k=1}^{\infty} \frac{A \mid Q^{*} \epsilon^{\epsilon / n}}{\left(2^{k}\left|Q^{*}\right|^{1 / n}\right)^{m n+\epsilon}} \int_{\left(2^{k} Q^{*}\right)^{h}} \prod_{j=1}^{h}\left\|f_{j}\right\|_{\ell^{q_{j}}} d y_{j}\right. \\
& \left.+\sum_{k=1}^{\infty} \frac{A}{\left(2^{k}\left|Q^{*}\right|^{1 / n}\right)^{m n}} \int_{\left(2^{k} Q^{*}\right)^{h}} \prod_{j=1}^{h}\left\|f_{j}\right\|_{\ell^{q_{j}}} d y_{j}\right) \prod_{j=h+1}^{m} \int_{Q^{*}}\left\|f_{j}\right\|_{\ell^{q_{j}}} d y_{j} \\
\leq & C \sum_{k=0}^{\infty} \prod_{j=1}^{m} \frac{1}{2^{k \epsilon}} \frac{1}{\left(2^{k+1}\left|Q^{*}\right|^{1 / n}\right)^{n}} \int_{2^{k+1} Q^{*}}\left\|f_{j}\right\|_{\ell^{q_{j}}} d y_{j} \\
& +C \sum_{k=0}^{\infty} \frac{1}{2^{k n(m-h)}} \prod_{j=h+1}^{m} \frac{1}{\left|Q^{*}\right|} \int_{Q^{*}}\left\|f_{j}\right\|_{\ell^{q_{j}}} d y_{j} \\
& \times \prod_{j=1}^{h} \frac{1}{\left(2^{k+1}\left|Q^{*}\right|^{1 / n}\right)^{n}} \int_{2^{k+1} Q^{*}}\left\|f_{j}\right\|_{\ell^{q_{j}}} d y_{j} \\
\leq & C \mathcal{M}\left(\|\vec{f}\|_{\ell q}\right)(x)+\mathcal{M}_{\rho}\left(\|\vec{f}\|_{\ell q}\right)(x) .
\end{aligned}
$$

By a similar argument, we deduce that $I_{\infty, \ldots, 0}^{3} \leq C \mathcal{M}\left(\|\vec{f}\|_{\ell q}\right)(x)+\mathcal{M}_{\rho}\left(\|\vec{f}\|_{\ell q}\right)(x)$ and $I I_{\infty, \ldots, 0}^{2} \leq C \mathcal{M}\left(\|\vec{f}\|_{\ell q}\right)(x)$. 
Given any positive integer $m, \forall 1 \leq j \leq m$, let $C_{j}^{m}$ denote the family of all finite subset $\sigma=\{\sigma(1), \ldots, \sigma(j)\}$ of $j$ different elements. For any $\sigma \in C_{j}^{m}$ we associate the complementary sequence $\sigma^{\prime}$ given by $\sigma^{\prime}=\{1,2, \ldots, m\} \backslash \sigma$.

Lemma 15 Let $0<\delta<\epsilon<1 / m, 1 / m<q<\infty$, and $\frac{1}{q}=\frac{1}{q_{1}}+\cdots+\frac{1}{q_{m}}$ with $1<q_{1}, \ldots, q_{m}<\infty$. Suppose that $\vec{b} \in(B M O)^{\ell}$. Then there exists a constant $C>0$ depending only on $\delta$ and $\epsilon$ such that

$$
\begin{aligned}
M_{\delta}^{\sharp}\left(T_{\Pi \vec{b}, q} \vec{f}\right)(x) \leq & C \prod_{j=1}^{\ell}\left\|b_{j}\right\|_{B M O}\left(\mathcal{M}_{L(\log L)}\|\vec{f}\|_{\ell^{q}}(x)+M_{\epsilon}\left(T_{q}(\vec{f})\right)(x)\right) \\
& +C \sum_{i=1}^{\ell-1} \sum_{\sigma \in C_{i}^{\ell}} C_{i, \ell} \prod_{j \in \sigma}\left\|b_{j}\right\|_{B M O} M_{\epsilon}\left(T_{\Pi \vec{b}_{\sigma^{\prime}}, q^{f}} \vec{f}\right)(x)
\end{aligned}
$$

for any smooth vector function $\left\{f_{k}\right\}_{k=1}^{\infty}$ for any $x \in \mathbb{R}^{n}$.

Proof For simplicity of notation, we replace $\prod_{j=1}^{m} f_{j}\left(y_{j}\right)$ by $F(\vec{y})$ and let $\lambda_{j}=\frac{1}{2|Q|} \int_{2 Q} b_{j}(z) d z$, for $j=1, \ldots, \ell$. Let $x \in \mathbb{R}^{n}$ and $Q$ be a cube centered at $x$. We have

$$
\begin{aligned}
T_{\Pi \vec{b}}(\vec{f})(x)= & \left(\int_{0}^{\infty}\left|\int_{\left(\mathbb{R}^{n}\right)^{m}} \prod_{j=1}^{\ell}\left(b_{j}(x)-b_{j}\left(y_{j}\right)\right) K_{v}(x, \vec{y}) F(\vec{y}) d \vec{y}\right|^{2} \frac{d v}{v}\right)^{1 / 2} \\
= & \left(\int_{0}^{\infty}\left|\int_{\left(\mathbb{R}^{n}\right)^{m}} \prod_{j=1}^{\ell}\left(\left(b_{j}(x)-\lambda_{j}\right)-\left(b_{j}\left(y_{j}\right)-\lambda_{j}\right)\right) K_{v}(x, \vec{y}) F(\vec{y}) d \vec{y}\right|^{2} \frac{d v}{v}\right)^{1 / 2} \\
\leq & \sum_{i=0}^{\ell} \sum_{\sigma \in C_{i}^{\ell}} \prod_{j \in \sigma}\left|b_{j}(x)-\lambda_{j}\right| \\
& \times\left(\int_{0}^{\infty}\left|\int_{\left(\mathbb{R}^{n}\right)^{m}} \prod_{j \in \sigma^{\prime}}\left(b_{j}\left(y_{j}\right)-\lambda_{j}\right) K_{v}(x, \vec{y}) F(\vec{y}) d \vec{y}\right|^{2} \frac{d v}{v}\right)^{1 / 2} \\
= & \prod_{j=1}^{\ell}\left|b_{j}(x)-\lambda_{j}\right| T(\vec{f})(x)+T\left(\prod_{j=1}^{\ell}\left(b_{j}(\cdot \cdot j)-\lambda_{j}\right) \vec{f}\right)^{(x)} \\
& +\sum_{i=1}^{\ell-1} \sum_{\sigma \in C_{i}^{\ell}} \prod_{j \in \sigma}\left|b_{j}(x)-\lambda_{j}\right| \\
& \times\left(\int_{0}^{\infty}\left|\int_{\left(\mathbb{R}^{n}\right)^{m}} \prod_{j \in \sigma^{\prime}}\left(b_{j}\left(y_{j}\right)-\lambda_{j}\right) K_{v}(x, \vec{y}) F(\vec{y}) d \vec{y}\right|^{2} \frac{d v}{v}\right)^{1 / 2} .
\end{aligned}
$$

Noting that $b_{j}\left(y_{j}\right)-\lambda_{j}=\left(b_{j}\left(y_{j}\right)-b_{j}(x)\right)+\left(b_{j}(x)-\lambda_{j}\right)$, we get

$$
\begin{aligned}
T_{\Pi \vec{b}, q}(\vec{f})(z)= & \prod_{j=1}^{\ell}\left|b_{j}(z)-\lambda_{j}\right| T_{q}(\vec{f})(z)+T_{q}\left(\prod_{j=1}^{\ell}\left(b_{j}(\cdot j)-\lambda_{j}\right) \vec{f}\right)(z) \\
& +\sum_{i=1}^{\ell-1} \sum_{\sigma \in C_{i}^{\ell}} C_{i, \ell} \prod_{j \in \sigma}\left|b_{j}(z)-\lambda_{j}\right| T_{\Pi \vec{b}_{\sigma^{\prime}, q}} \vec{f}(z) .
\end{aligned}
$$

Here $C_{i, \ell}$ depends only on $i$ and $\ell$. 
Let $c_{0}=\|c\|_{\ell q}=\left(\sum_{k=1}^{\infty}\left|c_{k}\right|^{q}\right)^{1 / q}$. Since $0<\delta<1 / m<1$, we have

$$
\begin{aligned}
\left(\left.\frac{1}{|Q|} \int_{Q}|| T_{\Pi \vec{b}, q} \vec{f}(z)\right|^{\delta}-\left|c_{0}\right|^{\delta} \mid d z\right)^{1 / \delta} & \\
\leq & C\left(\frac{1}{|Q|} \int_{Q}\left\|T_{\Pi \vec{b}}(\vec{f})(z)-c\right\|_{\ell^{q}}^{\delta} d z\right)^{1 / \delta} \\
\leq & C\left(\frac{1}{|Q|} \int_{Q}\left\|\prod_{j=1}^{\ell}\left|b_{j}(x)-\lambda_{j}\right| T(\vec{f})(z)\right\|_{\ell^{q}}^{\delta} d z\right)^{1 / \delta} \\
& +C \sum_{i=1}^{\ell-1} \sum_{\sigma \in C_{i}^{\ell}} C_{i, \ell}\left(\frac { 1 } { | Q | } \int _ { Q } \prod _ { j \in \sigma } \left(\left|b_{j}(z)-\lambda_{j}\right| T_{\left.\left.\Pi \overrightarrow{b_{\sigma^{\prime}}, q}, \vec{f}(z)\right)^{\delta} d z\right)^{1 / \delta}}\right.\right. \\
& +C\left(\frac{1}{|Q|} \int_{Q}\left\|T\left(\prod_{j=1}^{\ell}\left(b_{j}\left(\cdot \cdot_{j}\right)-\lambda_{j}\right) \vec{f}\right)(z)-c\right\|_{\ell q}^{\delta} d z\right)^{1 / \delta} \\
:= & I+I I+I I I .
\end{aligned}
$$

Now exploiting the standard Hölder inequality for finitely many functions with $1<p<$ $\epsilon / \delta$, it follows that

$$
\begin{aligned}
& I \leq C \prod_{j=1}^{\ell}\left\|b_{j}\right\|_{B M O} M_{\epsilon}\left(T_{q} \vec{f}\right)(x), \\
& I I \leq C \sum_{i=1}^{\ell-1} \sum_{\sigma \in C_{i}^{\ell}} C_{j, \ell} \prod_{j \in \sigma}\left\|b_{j}\right\|_{B M O} M_{\epsilon}\left(T_{\Pi \vec{b}} \vec{f}\right)(x) .
\end{aligned}
$$

Next let us address part III. Set $\vec{f}_{j}=\vec{f}_{j}^{0}+\vec{f}_{j}^{\infty}$, where $\vec{f}_{j}^{0}=\vec{f}_{j} \chi_{Q^{*}}$. Let $\vec{f}^{\alpha}=f_{1}^{\alpha_{1}} \cdots f_{m}^{\alpha_{m}}$ and $Q^{*}=(8 \sqrt{n}+4) Q$. Taking $c_{0}=\sum_{\alpha_{1}, \ldots, \alpha_{m}}\left\|T\left(\left(b_{1}\left(\cdot_{1}\right)-\lambda_{1}\right) \cdots\left(b_{\ell}(\cdot \ell)-\lambda_{\ell}\right)\right) f_{1}^{\alpha_{1}} \cdots f_{m}^{\alpha_{m}}(x)\right\|_{\ell q}$, we have

$$
\begin{aligned}
\| & T\left(\prod_{j=1}^{\ell}\left(b_{j}\left(\cdot \cdot_{j}\right)-\lambda_{j}\right) \vec{f}\right)(z)-c \|_{\ell q} \\
\leq & \left|T_{q}\left(\prod_{j=1}^{\ell}\left(b_{j}\left(\cdot \cdot_{j}\right)-\lambda_{j}\right) \vec{f}^{0}\right)(z)\right| \\
& +C \sum_{\alpha_{1}, \ldots, \alpha_{m}}\left\|T\left(\prod_{j=1}^{\ell}\left(b_{j}\left(\cdot \cdot_{j}\right)-\lambda_{j}\right) \vec{f}^{\alpha}\right)(z)-T\left(\prod_{j=1}^{\ell}\left(b_{j}\left(\cdot \cdot_{j}\right)-\lambda_{j}\right) \vec{f}^{\alpha}\right)(x)\right\|_{\ell q},
\end{aligned}
$$

where in the last sum each $\alpha_{j}=0$ or $\infty$ and in each term there is at least one $\alpha_{j}=\infty$.

If $\alpha_{1}=\cdots=\alpha_{m}=0$, Kolmogorov's inequality and Theorem 6 imply

$$
\begin{aligned}
& \left(\frac{1}{|Q|}\left|T_{q}\left(\prod_{j=1}^{\ell}\left(b_{j}\left(\cdot \cdot_{j}\right)-\lambda_{j}\right) \vec{f}^{0}\right)(z)\right|^{\delta}\right)^{1 / \delta} \\
& \leq C\left\|T_{q}\left(\prod_{j=1}^{\ell}\left(b_{j}(\cdot j)-\lambda_{j}\right) \vec{f}^{0}\right)\right\|_{L^{1 / m, \infty}\left(Q, \frac{d z}{|Q|}\right)}
\end{aligned}
$$




$$
\begin{aligned}
& \leq C \prod_{j=1}^{\ell}\left\|b_{j}\right\|_{B M O}\|\| f_{j}\left\|_{\ell^{q_{j}}}\right\|_{L(\log L), Q} \prod_{j=\ell+1}^{m} \frac{1}{|Q|} \int_{Q}\left\|f_{j}\right\|_{\ell^{q_{j}}} d z \\
& \leq C \prod_{j=1}^{\ell}\left\|b_{j}\right\|_{B M O} \mathcal{M}_{L(\log L)}\left(\left\|f_{j}\right\|_{\ell^{q_{j}}}\right)(x) .
\end{aligned}
$$

If $\alpha_{1}=\cdots=\alpha_{m}=\infty$, applying Hölder's inequality and Minkowski's inequality, then we get

$$
\begin{aligned}
& \left(\frac{1}{|Q|} \int_{Q}\left\|T\left(\prod_{j=1}^{\ell}\left(b_{j}\left(\cdot_{j}\right)-\lambda_{j}\right) \vec{f}^{\alpha}\right)(z)-T\left(\prod_{j=1}^{\ell}\left(b_{j}\left(y_{j}\right)-\lambda_{j}\right) \vec{f}^{\alpha}\right)(x)\right\|_{\ell q}^{\delta}\right)^{1 / \delta} \\
& \leq \frac{C}{|Q|} \int_{Q}\left(\sum _ { k = 1 } ^ { \infty } \left(\int_{0}^{\infty}\left|\int_{\left(\mathbb{R}^{n} \backslash Q^{*}\right)^{m}}\right| K_{v}(z, \vec{y})-K_{v}(x, \vec{y}) \mid\right.\right. \\
& \left.\left.\times\left.\prod_{j=1}^{\ell}\left(b_{j}\left(y_{j}\right)-\lambda_{j}\right) f_{1 k}\left(y_{1}\right) \cdots f_{m k}\left(y_{m}\right) d \vec{y}\right|^{2} \frac{d v}{v}\right)^{q / 2}\right)^{1 / q} d z \\
& \leq \frac{C}{|Q|} \int_{Q}\left(\sum_{k=1}^{\infty} \mid \int_{\left(\mathbb{R}^{n} \backslash Q^{*}\right)^{m}}\left(\int_{0}^{\infty}\left|K_{\nu}(z, \vec{y})-K_{\nu}(x, \vec{y})\right|^{2} \frac{d v}{v}\right)^{1 / 2}\right. \\
& \left.\times\left.\prod_{j=1}^{\ell}\left(b_{j}\left(y_{j}\right)-\lambda_{j}\right) f_{1 k}\left(y_{1}\right) \cdots f_{m k}\left(y_{m}\right) d \vec{y}\right|^{q}\right)^{1 / q} d z \\
& \leq \frac{C}{|Q|} \int_{Q}\left(\sum_{k=1}^{\infty} \mid \int_{\left(\mathbb{R}^{n} \backslash Q^{*}\right)^{m}}\left(\int_{0}^{\infty}\left|K_{\nu}(z, \vec{y})-K_{v, t}^{0}(z, \vec{y})\right|^{2} \frac{d v}{v}\right)^{1 / 2}\right. \\
& \left.\times\left.\prod_{j=1}^{\ell}\left(b_{j}\left(y_{j}\right)-\lambda_{j}\right) f_{1 k}\left(y_{1}\right) \cdots f_{m k}\left(y_{m}\right) d \vec{y}\right|^{q}\right)^{1 / q} d z \\
& +\frac{C}{|Q|} \int_{Q}\left(\sum_{k=1}^{\infty} \mid \int_{\left(\mathbb{R}^{n} \backslash Q^{*}\right)^{m}}\left(\int_{0}^{\infty}\left|K_{v, t}^{0}(z, \vec{y})-K_{v, t}^{0}(x, \vec{y})\right|^{2} \frac{d v}{v}\right)^{1 / 2}\right. \\
& \left.\times\left.\prod_{j=1}^{\ell}\left(b_{j}\left(y_{j}\right)-\lambda_{j}\right) f_{1 k}\left(y_{1}\right) \cdots f_{m k}\left(y_{m}\right) d \vec{y}\right|^{q}\right)^{1 / q} d z \\
& +\frac{C}{|Q|} \int_{Q}\left(\sum_{k=1}^{\infty} \mid \int_{\left(\mathbb{R}^{n} \backslash Q^{*}\right)^{m}}\left(\int_{0}^{\infty}\left|K_{v, t}^{0}(x, \vec{y})-K_{v}(x, \vec{y})\right|^{2} \frac{d v}{v}\right)^{1 / 2}\right. \\
& \left.\times\left.\prod_{j=1}^{\ell}\left(b_{j}\left(y_{j}\right)-\lambda_{j}\right) f_{1 k}\left(y_{1}\right) \cdots f_{m k}\left(y_{m}\right) d \vec{y}\right|^{q}\right)^{1 / q} d z \\
& :=I I I_{1}+I I I_{2}+I I I_{3} \text {. }
\end{aligned}
$$

First we consider $I I I_{1}$. Taking $t=[2 \sqrt{n} \ell(Q)]^{s}$, by Assumption $(\mathrm{H} 2)$ we have

$$
\begin{aligned}
I I I_{1} & \leq \frac{C}{|Q|} \int_{Q} \int_{\left(\mathbb{R}^{n} \backslash Q^{*}\right)^{m}} \frac{A t^{\epsilon / s}}{\left(\sum_{j=1}^{m}\left|z-y_{j}\right|\right)^{m n+\epsilon}} \prod_{j=1}^{\ell}\left|b_{j}\left(y_{j}\right)-\lambda_{j}\right|\left\|f_{1}\right\|_{\ell q_{1}} \cdots\left\|f_{m}\right\|_{\ell q_{m}} d \vec{y} d z \\
& \leq C \sum_{k=1}^{\infty} \frac{1}{2^{k \epsilon}} \prod_{j=1}^{\ell} \frac{1}{2^{(k+1) n}\left|Q^{*}\right|} \int_{2^{k+1} Q^{*}}\left|b_{j}\left(y_{j}\right)-\lambda_{j}\right|\left\|f_{j}\right\|_{\ell^{q_{j}}} d y_{j}
\end{aligned}
$$




$$
\begin{aligned}
& \times \prod_{j=\ell+1}^{m} \frac{1}{2^{(k+1) n}\left|Q^{*}\right|} \int_{2^{k+1} Q^{*}}\left\|f_{j}\right\|_{\ell^{q_{j}}} d y_{j} \\
\leq & C \sum_{k=1}^{\infty} \frac{1}{2^{k \epsilon}} \prod_{j=1}^{\ell}\left\|b_{j}\right\|_{B M O}\|\| f_{j}\left\|_{\ell^{q_{j}}}\right\|_{L(\log L), 2^{k+1} Q^{*}} \\
& \times \prod_{j=\ell+1}^{m} \frac{1}{2^{(k+1) n}\left|Q^{*}\right|} \int_{2^{k+1} Q^{*}}\left\|f_{j}\right\|_{\ell^{q_{j}}} d y_{j} \\
\leq & C \mathcal{M}_{L(\log L)}\left(\|\vec{f}\|_{\ell q}\right)(x) .
\end{aligned}
$$

Similarly, we have $I I I_{2} \leq C \mathcal{M}_{L(\log L)}\left(\|\vec{f}\|_{\ell q}\right)(x)$ and $I I I_{3} \leq C \mathcal{M}_{L(\log L)}\left(\|\vec{f}\|_{\ell q}\right)(x)$. Now it remains to consider the typical case of $I I I$,

$$
\begin{aligned}
&\left(\frac{1}{|Q|} \int_{Q} \|\right. T\left(\prod_{j=1}^{\ell}\left(b_{j}(\cdot j)-\lambda_{j}\right) f_{1}^{\infty}, \ldots, f_{\ell}^{\infty}, f_{\ell+1}^{0}, \ldots, f_{m}^{0}\right)(z) \\
&\left.-\left.T\left(\prod_{j=1}^{\ell}\left(b_{j}\left(y_{j}\right)-\lambda_{j}\right) f_{1}^{\infty}, \ldots, f_{\ell}^{\infty}, f_{\ell+1}^{0}, \ldots, f_{m}^{0}\right)(x)\right|_{\ell q} ^{\delta}\right)^{1 / \delta} \\
& \leq \frac{C}{|Q|} \int_{Q}\left(\sum _ { k = 1 } ^ { \infty } \left(\int_{0}^{\infty}\left|\int_{\left(\mathbb{R}^{n} \backslash Q^{*}\right)^{m}}\right| K_{v}(z, \vec{y})-K_{v}(x, \vec{y}) \mid \prod_{j=1}^{\ell}\left(b_{j}\left(y_{j}\right)-\lambda_{j}\right)\right.\right. \\
&\left.\left.\quad \times\left. f_{1 k}^{\infty}\left(y_{1}\right) \cdots f_{\ell k}^{\infty}\left(y_{\ell}\right) f_{\ell+1, k}^{0}\left(y_{\ell+1}\right) \cdots f_{m k}^{0}\left(y_{m}\right) d \vec{y}\right|^{2} \frac{d v}{v}\right)^{q / 2}\right)^{1 / q} d z \\
& \leq\left(\int_{\left(\mathbb{R}^{n} \backslash Q^{*}\right)^{\ell}} \frac{t^{\epsilon / s} \prod_{j=1}^{\ell}\left(b_{j}\left(y_{j}\right)-\lambda_{j}\right)\left\|f_{j}\left(y_{j}\right)\right\|_{\ell^{q_{j}}} d y_{j}}{\left(\sum_{j=1}^{\ell}\left|z-y_{j}\right|\right)^{m n+\epsilon}}\right. \\
&\left.\quad+\int_{\left(\mathbb{R}^{n} \backslash Q^{*}\right)^{\ell}} \frac{\prod_{j=1}^{\ell}\left(b_{j}\left(y_{j}\right)-\lambda_{j}\right)\left\|f_{j}\left(y_{j}\right)\right\|_{\ell^{q_{j}}} d y_{j}}{\left(\sum_{j=1}^{\ell}\left|z-y_{j}\right|\right)^{m n}}\right) \prod_{j=\ell+1}^{m} \int_{Q^{*}}\left\|f\left(y_{j}\right)\right\|_{\ell^{q_{j}}} d y_{j} \\
& \leq C \prod_{j=1}^{\ell}\left\|b_{j}\right\|_{B M O} \mathcal{M}_{L(\log L)}\left(\|\vec{f}\|_{\ell q}\right)(x) .
\end{aligned}
$$

Lemma 16 Let $0<p<\infty, 1 / m<q<\infty$, and $\frac{1}{q}=\frac{1}{q_{1}}+\cdots+\frac{1}{q_{m}}$ with $1<q_{1}, \ldots, q_{m}<\infty$ and let $\omega \in A_{\infty}$. Suppose that $\vec{b} \in(B M O)^{\ell}$. Then there exists a constant $C>0$ such that

$$
\int_{\mathbb{R}^{n}}\left|T_{\Pi \vec{b}, q} \vec{f}\right|^{p} \omega(x) d x \leq C \prod_{j=1}^{\ell}\left\|b_{j}\right\|_{B M O}^{p} \int_{\mathbb{R}^{n}}\left(\mathcal{M}_{L(\log L)}\left(\|\vec{f}\|_{\ell q}\right)(x)\right)^{p} \omega(x) d x
$$

The proof is similar to [16], so we omit it here.

Based on the above lemmas, the proof of Theorem 1.3 in [16] provides the main ideas for the proof of Theorem 7 .

The authors declare that they have no competing interests. 


\section{Acknowledgements}

The first author was supported by the National Natural Science Foundation of China (No. 11401175). The second author was supported by the Fundamental Research Funds for the Central Universities (No. 3102015ZY068). The authors thank the referees for carefully reading the manuscript and providing many valuable suggestions, which have improved the article.

Received: 15 October 2015 Accepted: 26 November 2015 Published online: 14 December 2015

\section{References}

1. Xue, $Q$, Yan, J: On multilinear square function and its application to multilinear Littlewood-Paley operators with non-convolution type kernels. J. Math. Anal. Appl. 422, 1342-1362 (2015)

2. Hormozi, M, Si, Z, Xue, Q: On general multilinear square function with non-smooth kernels (2015). arXiv:1506.08922 [math.CA]

3. Coifman, RR, McIntosh, A, Meyer, Y: Lintegrale de Cauchy definit un operateur borne sur $L^{2}$ pour les courbes Lipschitziennes. Ann. Math. 116, 361-387 (1982)

4. Coifman, RR, Deng, D, Meyer, Y: Domains de la racine carrée de certains opérateurs différentiels accrétifs. Ann. Inst. Fourier (Grenoble) 33, 123-134 (1983)

5. David, G, Journe, JL: Une caracterisation des operateurs integraux singuliers bornes sur $L^{2}\left(\mathbb{R}^{n}\right)$. C. R. Math. Acad. Sci. Paris 296, 761-764 (1983)

6. Fabes, EB, Jerison, D, Kenig, C: Multilinear Littlewood-Paley estimates with applications to partial differential equations. Proc. Natl. Acad. Sci. 79, 5746-5750 (1982)

7. Fabes, EB, Jerison, D, Kenig, C: Necessary and sufficient conditions for absolute continuity of elliptic harmonic measure. Ann. Math. 119, 121-141 (1984)

8. Fabes, EB, Jerison, D, Kenig, C: Multilinear square functions and partial differential equations. Am. J. Math. 107, $1325-1368$ (1985)

9. Xue, Q, Peng, X, Yabuta, K: On the theory of multilinear Littlewood-Paley g-function. J. Math. Soc. Jpn. 67, 535-559 (2015)

10. Lerner, AK, Ombrosi, S, Pérez, C, Torres, RH, Trujillo-González, R: New maximal functions and multiple weights for the multilinear Calderón-Zygmund theory. Adv. Math. 220, 1222-1264 (2009)

11. Anh, BT, Duong, XT: On commutators of vector $B M O$ functions and multilinear singular integrals with non-smooth kernels. J. Math. Anal. Appl. 371, 80-84 (2010)

12. Grafakos, L, Martell, JM: Extrapolation of weighted norm inequalities for multivariable operators. J. Geom. Anal. 14 19-46 (2004)

13. Fefferman, C, Stein, EM: $H^{p}$ spaces of several variables. Acta Math. 129, 173-193 (1972)

14. Cruz-Uribe, D, Martell, JM, Pérez, C: Extrapolation from $A_{\infty}$ weights and applications. J. Funct. Anal. 213, 412-439 (2004)

15. Andersen, KF, John, RT: Weighted inequalities for vector-valued maximal functions and singular integrals. Stud. Math. 69, 19-31 (1980)

16. Si, Z: Weighted estimates for vector-valued multilinear operators with non-smooth kernels. J. Inequal. Appl. 2013, 250 (2013)

\section{Submit your manuscript to a SpringerOpen ${ }^{\ominus}$ journal and benefit from:}

- Convenient online submission

- Rigorous peer review

Immediate publication on acceptance

- Open access: articles freely available online

- High visibility within the field

- Retaining the copyright to your article 